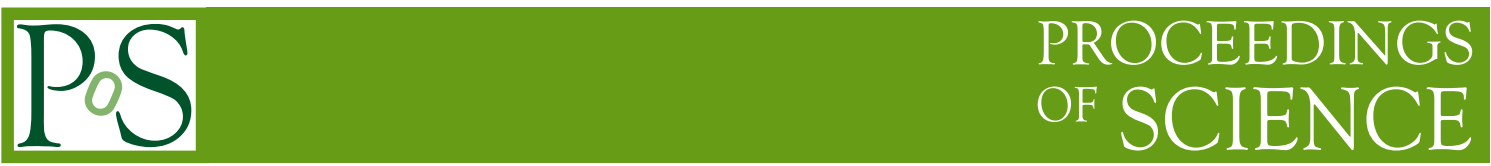

\title{
Search of New Physics with Kaon decays at NA62
}

\author{
Gianmaria COLLAZUOL*广 \\ Scuola Normale Superiore and INFN Pisa \\ E-mail: gianmaria.collazuolepi.infn.it
}

NA62 is a fixed target experiment at the CERN SPS aiming at the search for new phenomena beyond the Standard Model (SM) by measuring two of the theoretically most clean processes in kaon decays. Two phases can be distinguished in the NA62 physics programme. During the current short term phase (data taking completed in years 2007-8) we are studying the ratio $R_{K}=\frac{\Gamma\left(K \rightarrow e v_{e}(\gamma)\right)}{\Gamma\left(K \rightarrow \mu v_{\mu}(\gamma)\right)}$ of leptonic decay rates, which provides a golden probe for testing the structure of the weak interactions. In the second phase (long term) we will then focus on the measurement of the branching ratio of the very rare kaon decay $K^{+} \rightarrow \pi^{+} v \bar{v}$ aiming at collecting $O(100)$ $K^{+} \rightarrow \pi^{+} v \bar{v}$ events with $10 \%$ background in two years of data taking.

In this paper we first summarize the status of the $R_{K}$ analysis, based on $40 \%$ of the 2007 NA62 data sample, and discuss the preliminary result $R_{K}=(2.500 \pm 0.016) 10^{-5}$, which breaks for the first time the $1 \%$ error level and is consistent with the Standard Model. A status report of the longer term project, recently approved at CERN, will then follow, including the current R\&D programme and future perspectives for the $K^{+} \rightarrow \pi^{+} v \bar{v}$ measurement, for which data taking will start in year 2012.

European Physical Society Europhysics Conference on High Energy Physics July 16-22, 2009

Krakow, Poland

\footnotetext{
* Speaker.

${ }^{\dagger}$ On behalf of the NA62 collaboration
} 


\section{Introduction}

In the Standard Model of Particle Physics (SM) sub-permille accuracy is reached in the prediction of the ratios $R_{P}=\frac{\Gamma\left(P \rightarrow e v_{e}(\gamma)\right)}{\Gamma\left(P \rightarrow \mu v_{\mu}(\gamma)\right)}=\left(\frac{m_{e}}{m_{\mu}}\right)^{2}\left(\frac{m_{P}^{2}-m_{e}^{2}}{m_{P}^{2}-m_{\mu}^{2}}\right)^{2}\left(1+\delta R_{\mathrm{QED}}\right)$ of decay rates for the pseudoscalar $K$ and $\pi$ mesons. This excellent accuracy $\left(R_{K}=2.477 \pm 0.001 \times 10^{-5}, R_{\pi}=12.352 \pm\right.$ $\left.0.001 \times 10^{-5}\right)$ is due to cancelations of the hadronic uncertainties $[1,2]$ and to the precise knowledge of the electromagnetic correction $\delta R_{\mathrm{QED}}$. The factor $\left(m_{e} / m_{\mu}\right)^{2}$ accounts for helicity suppression and make $R_{P}$ very sensitive to SM extensions involving Pseudo-Scalar currents and nonuniversal corrections, which are expected to induce variations of $R_{P}$ of the order $10^{-4}<\delta R_{P} / R_{P}<$ $10^{-2}$. In particular SM extensions involving Lepton Flavour Violation (LFV) are currently not ruled out by experiment. The current world average of measurements is dominated by recent preliminary results from NA48/2 [3] and KLOE [4], whose combined result is limited at a precision level of $1 \%$. The aim of NA62 (short term phase [5]) is to measure $R_{K}$ reaching a new accuracy level $<0.4 \%$. To this purpose, a data taking strategy allowing control over the systematic effects, and in particular precise background subtraction, was worked out, and a data sample of $\sim 0.15 \times 10^{6} K_{e 2}$ candidates was collected with a background level below $\sim 10 \%$.

While leptonic kaon decays are a golden opportunity for studying new physics (NP) effects involved in MFV scenarios, $K \rightarrow \pi \nu \bar{v}$ decays, on the other hand, give unique and clean information about the flavour structure of the $s \rightarrow d$ sector in any model with new degrees of freedom in the $\mathrm{TeV}$ range. Sizable deviations from SM are expected in a variety of models [6]. Also, in some specific scenarios NP effects could be seen in $K \rightarrow \pi v \bar{v}$ decays even without any new particles seen at LHC or even without significant signals in $\mathrm{B}_{\mathrm{d}, \mathrm{s}}$ decays. The flavor changing neutral current $K^{+} \rightarrow \pi^{+} v \bar{v}$ process proceeds through box and purely electro-weak penguin diagrams and is very clean theoretically: short distance dynamics dominates, c-quarks contributions have been evaluated to NNLO order at $5 \%$, and the hadronic matrix elements can be parameterized in terms of the $K^{+} \rightarrow \pi^{0} e^{+} \bar{v}$ branching ratio that is well known experimentally. The computed branching ratio is $(0.85 \pm 0.07) \cdot 10^{-10}$. The existing measurement, based on 7 events from E787/949 experiments at BNL, is $\left(1.73_{-1.05}^{+1.15}\right) \cdot 10^{-10}$, compatible with the SM within errors. A $10 \%$ accuracy measurement is required to provide a significative test of NP scenarios.

\section{The $R_{K}$ measurement}

The NA48/2 beam line and setup were used; running conditions were optimized for the $\mathrm{Ke} 2$ measurement in 2007 using the experience of earlier studies based on NA48/2 data sets [3]. The beam line K12 at CERN SPS is capable of delivering simultaneous narrow momentum band $\mathrm{K}^{+}$ and $K^{-}$beams. Most of the data were taken with the $K^{+}$beam only because the muon sweeping system is more effective in rejecting $\mu^{+}$. Conversely $\sim 10 \%$ of the data were recorded with the $K^{-}$beam only in order to measure the background induced by the beam halo via $\mu \rightarrow e$ decays. A central momentum of $74 \mathrm{GeV} / \mathrm{c}$ was used in 2007 data taking. $K_{e 2}$ and $K_{\mu 2}$ events were collected simultaneously, in order the result do not rely on kaon flux measurement and several systematic effects, such as parts of the trigger and detection efficiencies, cancel in the ratio. The main data sample was taken during four months of running in 2007 corresponding to about $4 \times 10^{6}$ SPS spills. Additional data were collected in 2008 for systematic studies. The main detectors used for the 
$R_{K}$ measurement include the NA48 liquid krypton ( $\mathrm{LKr}$ ) electromagnetic calorimeter $(\gamma$ detection and particle identification), the magnetic spectrometer $\left(\delta p_{[\mathrm{GeV} / \mathrm{c}]} / p=0.47 \% \oplus p \cdot 0.02 \%\right)$ and the scintillator hodoscope (timing measurement and fast, minimum bias trigger).

The analysis is performed independently in bins of momentum of the charged track $(15 \mathrm{GeV} / \mathrm{c}<$ $p<65 \mathrm{GeV} / \mathrm{c}$ ), due to strong dependence on this variable of backgrounds and acceptances. Due to the topological similarity of $K_{e 2}$ and $K_{\mu 2}$ decays, a large part of the selection criteria is common for both decays; this leads to cancelations of the related systematic uncertainties in $R_{K}$. The ratio $R_{K}$ in each momentum bin is computed after background subtraction. Correction factors are then applied in order to account for acceptances and for trigger, reconstruction and particle identification efficiencies. A highly efficient $K_{e 2}$ over $K_{\mu 2}$ discrimination with excellent rejection factor $\left(10^{6}\right)$ is obtained (a) by exploiting the ratio of the energy deposition in $\mathrm{LKr}$ and the momentum measured by the spectrometer and (b) by performing kinematic identification by means of the missing invariant mass. In the present analysis, based on the study of a sub-sample (40\%) of the full data sample, we identify $\sim 51 \times 10^{3} K_{e 2}$ and $\sim 16 \times 10^{6}$ and $K_{\mu 2}$ candidates respectively. Detailed studies with data and Monte Carlo samples are performed for measuring any source of background (bkg). The main contributions come from: (1) $K_{\mu 2}$ events where a $\mu$ is mis-identified as electron due to "catastrophic" bremsstrahlung in the calorimeter (bkg/signal at level of $8.07 \pm 0.21 \%$ ); (2) $K_{e 2 \gamma}$ events $(1.29 \pm 0.32 \%)$; (3) muons in the beam halo which decay to electrons $(1.23 \pm 0.07 \%)$.

The preliminary NA62 result $R_{K}=\left(2.500 \pm 0.012_{\text {stat. }} \pm 0.011_{\text {syst }}\right) \times 10^{-5}=(2.500 \pm 0.016) \times$ $10^{-5}$ is consistent with the SM expectation. Most of the residual systematic uncertainties come from the evaluation of background from $K_{e 2 \gamma}$ and from the systematics related to trigger dead-time, which will be investigated further in the future. The whole 2007Ü08 data sample will allow pushing the uncertainty of RK down to $0.4 \%$. The new world average for $R_{K}$ is $(2.498 \pm 0.014) \times 10^{-5}$.

\section{The $K^{+} \rightarrow \pi^{+} v \bar{v}$ measurement}

The long term phase of NA62 [7], recently approved by CERN SPSC and Research Board, aims at measuring $K^{+} \rightarrow \pi^{+} v \bar{v}$ decays with a sensitivity of $10^{-12}$ per event, by using the decayin-flight technique which allows a signal acceptance of $\sim 10 \%$. The goal is to observe $\sim 100$ signal events in two years of data taking with a background to signal ratio smaller than $10 \%$. The signature of the $K^{+} \rightarrow \pi^{+} v \bar{v}$ event is only one track in the initial and final states and nothing else. The main background sources consist of $K \rightarrow \pi \pi^{0}(63.5 \%)$ and $K \rightarrow \mu \nu$ (20.9\%) decays and background rejection relies on (1) precise timing for associating the outgoing $\pi$ to the correct incoming $K^{+}$; (2) kinematic rejection of two and three-body kaon decays; (3) $\mu$ and $\gamma$ vetoing; (4) particle identification for $K+/ \pi^{+}$and $\pi / \mu$ separation.

The $R \& D$ phase for the proposed experimental setup, described in the following, is well advanced and the final construction phase for some detectors has already started. In the proposed setup a high intensity $(800 \mathrm{MHz})$ and high momentum $(75 \mathrm{GeV} / \mathrm{c})$ unseparated beam of positively charged hadrons is produced by impinging the $400 \mathrm{GeV} / \mathrm{c}$ primary SPS protons beam onto a Be target, positrons being filtered out. The momentum and direction of each particle of the beam is measured by a beam spectrometer ("GigaTracKer") in vacuum. Kaons, being a fraction of $\sim 7 \%$, are tagged by a CEDAR differential Cerenkov counter. The "GigaTracKer" consists of three hybrid silicon micro-pixel tracking stations, covering an area of $60 \times 27 \mathrm{~mm}^{2}$ with pixels $300 \times 300 \mu \mathrm{m}^{2}$ 
wide and $200 \mu \mathrm{m}$ thick. A time resolution better than 200ps per station is required to provide a proper reconstruction of kaon tracks. The additional requirements of low material budget, radiation hardness and the need for operation in vacuum with an efficient cooling system make the "GigaTracKer" the main technological challenge of the experiment. Downsteam the beam spectrometer a high level vacuum $\left(<10^{-6}\right.$ mbar $)$ fiducial decay volume $(\sim 70 \mathrm{~m}$ in length $)$ is followed by a "pion" spectrometer, for measuring momenta and directions of kaon decay products. The "pion" spectrometer consists of low mass straw chambers in vacuum and is followed (downstream) by a $18 m$ long RICH detector filled with Ne at atmospheric pressure. The RICH provides pion identification $(15-35 \mathrm{GeV} / \mathrm{c}$ momentum range) and measures particle crossing times with a resolution better than $100 \mathrm{ps}$. Tests performed with a full length prototype indicate a $50 \mu \mathrm{rad}$ angle resolution and a 65 ps time resolution [8] in agreement with the design requirements. An almost hermetic set of calorimeters, with various shapes, including the existing NA48 LKr calorimeter and covering an azimuthal acceptance from $0 \mathrm{mrad}$ up to $50 \mathrm{mrad}$, will be used for vetoing photons produced in the decay region. Downstream the LKr calorimeter an active muon filter and veto will be located. The overall decay rate seen by the detectors downstream the decay volume will be $\sim 11 \mathrm{MHz}$, due to kaon decays and accidentals coming from the beam-line.

The sensitivity evaluation is based on the study of the missing invariant mass squared $\mathrm{m}_{\text {miss }}^{2}$ (with assumption of the pion mass). About $92 \%$ of the background is kinematically constrained (two-bodies decays) and can be rejected with a selection on $\mathrm{m}_{\text {miss. }}^{2}$. The residual $8 \%$ must be rejected by vetoes and particle identification. The required $\mu$ rejection (factor of $\sim 10^{7}$ ) is achieved by exploiting the different penetration of muons and pions through matter $\left(\sim 10^{5}\right)$, in addition to the RICH suppression $\left(\sim 10^{3}\right)$. The required photon rejection (factor $\sim 10^{8}$ ) is achieved by selecting pion with $p<35 \mathrm{GeV} / c$, so that for background from $\pi \pi^{0}$ decays at least $40 \mathrm{GeV}$ of energy is deposited in hermetic calorimeters and the $\pi^{0}$ can hardly be missed. The number of expected signal and background events for one year of data taking are respectively 55 and less than 8 , allowing a measurement of the $K^{+} \rightarrow \pi^{+} v \bar{v}$ branching ratio with $10 \%$ accuracy in two years of data taking.

\section{References}

[1] A. Masiero, P. Paradisi and R. Petronzio, Phys. Rev. D74 (2006) 011701.

[2] V. Cirigliano and I.Rosell, Phys. Lett. 99 (2007) 231801.

[3] Fiorini L., PoS (HEP2005) 288 2005; Kozhuharov V., PoS (KAON) 049 2007;

[4] F. Ambrosino et al., arXiv:0907.3594.

[5] NA48/2 and P-326 status report, CERN-SPSC-2006-033.

[6] M. Blanke, A. J. Buras, A. Poschenrieder, S. Recksiegel, C. Tarantino, S. Uhlig and A. Weiler, HEP-PH 0610298. JHEP 0701 (2007) 066.

[7] Anelli G. et al., CERN-SPSC-2005-013 and CERN-SPSC-P-326, 2005.

[8] G. Anzivino et al., Nucl. Instrum. Meth. A 593, 314 (2008). 